\title{
Application of the Acoustic Emission Technique to the Investigation of Fracture Processes in Glassy Polymers
}

\author{
Tetsuya NishiurA, ${ }^{*}$ Takashi JoH, ${ }^{*}$ Shogo OKUDA, ${ }^{* *}$ \\ and Masanobu MIKI** \\ *The Institute of Scientific and Industrial Research, Osaka University \\ Yamadakami, Suita, Osaka 565, Japan. \\ **Faculty of Domestic Science, Teikoku Women's University \\ Tohda-cho, Moriguchi, Osaka 575, Japan.
}

(Received June 3, 1980)

KEY WORDS Acoustic Emission / Fracture / Craze / Crack / Glassy
Polymer /

Recently, the acoustic emission (AE) technique has been widely applied to the investigation of fracture processes of metallic materials and composite materials such as fiber-reinforced plastics (FRP). ${ }^{1}$ However, there are only a few reports in which the $\mathrm{AE}$ analysis has been applied to the investigation of amorphous glassy polymers. ${ }^{2-4} \mathrm{AE}$ is a phenomena such that materials emit sound during the process of deformation and fracture which are accompanied by the release of strain energy. By detecting and analyzing $\mathrm{AE}$, some of the fundamental properties of deformation and fracture processes can be estimated.

It is well known that fracture processes in glassy polymers under tensile stress involve craze initiation, craze growth, crack initiation which takes place by craze break down, and crack growth. If $\mathrm{AE}$ can be detected in each fracture process, the $\mathrm{AE}$ technique will become a useful tool for clarifying the fracture mechanism of glassy polymers. The purpose of this study is to investigate the fracture mechanism of glassy polymers by the $\mathrm{AE}$ technique and also by the microscopic observation of the fracture process.

Commercially-available extruded poly(methyl methacrylate) (PMMA) sheets (Comoglas, Kyowa Gas Chem. Ind. Co. Ltd.) were used in this experiment, and tests were made at room temperature $\left(18-23^{\circ} \mathrm{C}\right)$. Tensile-test specimens were cut from a sheet of polymer so as to have the shape of a single-edge-notched specimen (length, $150 \mathrm{~mm}$; width, $30 \mathrm{~mm}$; thickness, $1 \mathrm{~mm}$; radius of notch root, $0.1 \mathrm{~mm}$ ). Figure 1 shows a block diagram of the apparatus for observing the $\mathrm{AE}$ of the polymers. Each specimen was loaded in tension with an Instron tensile machine (cross-head speed, $0.005 \mathrm{~cm} \mathrm{~min}^{-1}$, and the AE was simultaneously detected by means of two sensors (PZT transducers; resonance frequency, $140 \mathrm{kHz}$ ) fixed on the specimen. The AE signals were amplified by $80 \mathrm{~dB}$ with a preamlifier and a discriminator (NF Co. Ltd.); the signals rectified by the discriminator were recorded as the number of events by an electromagnetic oscillograph (visigraph). The craze zone (microcracks may be included) was continuously observed by an optical microscope, and photographed at definite intervals.

Two alcohols (methanol and ethanol) were used as crazing agents. The typical profiles of the craze zones for these alcohols are shown in Figure 2. The craze zones were different in each cases. The craze zones in the methanol immersion condition grew smoothly in a transverse direction throughout the specimen. In contrast to this, the growth of craze zones in ethanol was not smooth. As consistent with the difference in crazing behavior, AE could be observed only in ethanol.

Figure 3 shows the relation of loads, craze-zone growth profiles, and AE signals vs. strain of the crazing process of PMMA in ethanol. Slow growth of the craze zone was observed at the initial stage. When the strain was increased to a certain value, 


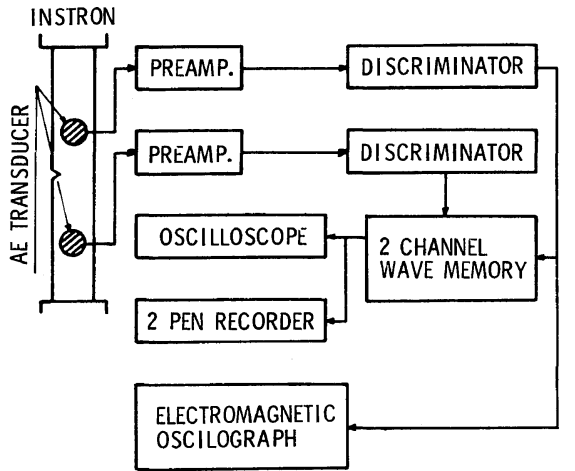

Figure 1. A block diagram of the apparatus for observing the acoustic emission of a polymer.

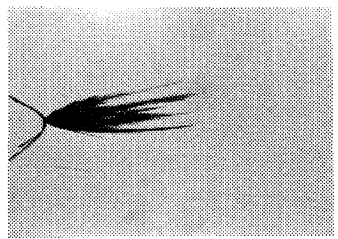

(a)

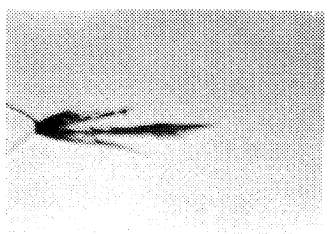

(b)
Figure 2. Profiles of craze zones formed in methanol (a) and in ethanol (b).

there was a rapid growth in the craze zone accompanied by strong AE signals. With further increase in the strain, a stepwise growth in the craze zone took place and AE signals could be detected along with the growth.

These results show that AE can be detected depending on the manner of growth of the craze zone. Thus, smooth growth of the craze zone yields AE signals that are not strong enough to be clearly separated from the noise level, but a jumpwise growth of the craze zone yields recordable $\mathrm{AE}$ signals.

These results differ somewhat from those reported by Roeder and Crostack. ${ }^{4}$ These authors indicated that AE in extruded PMMA could be detected by use of methanol as a crazing agent and that no change in the craze profile was observed before and after the initial AE sounds were produced. They also maintain that the transition from a craze to a crack can be traced by $\mathrm{AE}$ analysis. Our experiments suggest that AE signals

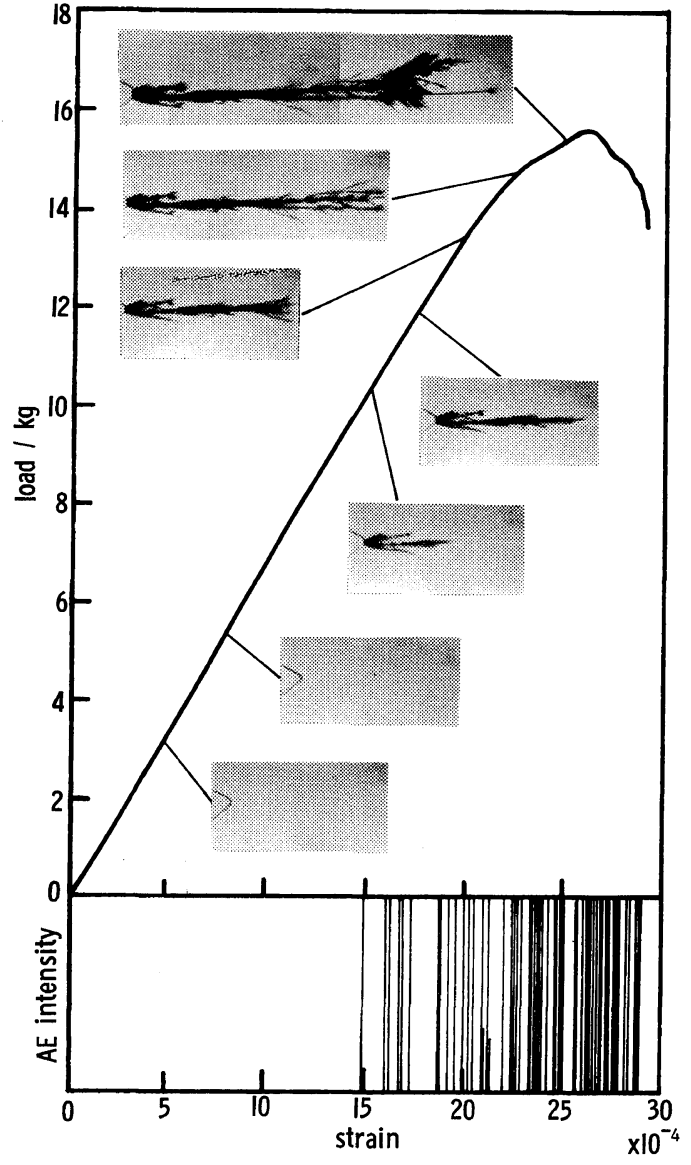

Figure 3. The relation of loads, profiles of craze-zone growth, and AE signals vs. strain for PMMA.

are not always produced in the transition from a craze to a crack. In order to clarify the process by which AE signals are produced, it is necessary to gather additional AE data and to analyze the emission characteristics in detail.

\section{REFERENCES}

1. R. G. Lipati, “Acoustic Emission," ASTM STP $\mathbf{5 0 5}$ (1972).

2. I. Grabec and A. Peterlin, J. Polym. Sci., Polym. Phys. Ed., 14, 651 (1976).

3. A. Peterlin, Adv. Chem. Ser., 174, 15 (1979), and references therein.

4. E. Roeder and H. A. Crostack, Kunststoffe, 67, 454 (1977). 